\title{
BMJ Open Ability of postoperative delirium to predict intermediate-term postoperative cognitive function in patients undergoing elective surgery at an academic medical centre: protocol for a prospective cohort study
}

\author{
Amrita Aranake-Chrisinger, ${ }^{1}$ Jenny Zhao Cheng, ${ }^{1}$ Maxwell R Muench, ${ }^{1,2}$ \\ Rose Tang, ${ }^{1}$ Angela Mickle, ${ }^{1}$ Hannah Maybrier, ${ }^{1}$ Nan Lin, ${ }^{1,3}$ Troy Wildes, \\ Eric Lenze, ${ }^{1}$ Michael Simon Avidan ${ }^{1}$
}

To cite: Aranake-Chrisinger A, Cheng JZ, Muench MR, et al. Ability of postoperative delirium to predict intermediate-term postoperative cognitive function in patients undergoing elective surgery at an academic medical centre: protocol for a prospective cohort study. BMJ Open 2018;8:e017079. doi:10.1136/ bmjopen-2017-017079

- Prepublication history and additional material for this paper are available online. To view these files, please visit the journal online (http://dx.doi. org/10.1136/bmjopen-2017017079).

Received 2 May 2017

Revised 17 0ctober 2017

Accepted 20 0ctober 2017

Check for updates

1Department of Anesthesiology, Washington University School of Medicine in St. Louis, Saint Louis, Missouri, USA

${ }^{2}$ Kirksville College of Osteopathic Medicine, Kirksville, Missouri, USA

${ }^{3}$ Division of Biostatistics, Department of Mathematics, Washington University in St. Louis, St. Louis, Missouri, USA

Correspondence to

Dr Amrita Aranake-Chrisinger; aranakea@wustl.edu

\section{ABSTRACT}

Introduction Postoperative delirium (POD) is a common complication in elderly patients, characterised by a fluctuating course of altered consciousness, disordered thinking and inattention. Preliminary research has linked POD with persistent cognitive impairment and decreased quality of life. However, these findings maybe confounded by patient comorbidities, postoperative complications and frailty. Our objective is to determine whether POD is an independent risk factor for persistent impairments in attention and executive function after elective surgery. Our central hypothesis is that patients with POD are more likely to have declines in cognition and quality of life 1 year after surgery compared with patients without POD. We aim to clarify whether these associations are independent of potentially confounding factors. We will also explore the association between POD and incident dementia.

Methods and analysis This study will recruit 200 patients from the ongoing Electroencephalography Guidance of Anesthesia to Alleviate Geriatric Syndromes (ENGAGES) study. Patients who live $\leq 45$ miles from the study centre or have a planned visit to the centre 10-16 months postoperatively will be eligible. Patients with POD, measured by the Confusion Assessment Method, will be compared with patients without delirium. The primary outcome of cognitive function and secondary outcomes of quality of life and incident dementia will be compared between cohorts. Cognition will be measured by Trails $A$ and $B$ and Stroop Color and Word Test, quality of life with Veteran's RAND 12-item Health Survey and incident dementia with the Short Blessed Test. Multivariable regression analyses and a Cox proportional hazards analysis will be performed. All results will be reported with $95 \%$ Cls and $\alpha=0.05$

Ethics and dissemination The study has been approved by the Washington University in St. Louis Institutional Review Board (IRB no 201601099). Plans for dissemination include scientific publications and presentations at scientific conferences.

Trial registration number NCT02241655.
Strengths and limitations of this study

- Postoperative intermediate term cognitive function and quality of life are important patient-centred outcomes.

- Analysis will include salient patient characteristics, including preoperative cognition, comorbid conditions and frailty.

- Study sample will consist of an understudied population in clinical research.

- Potential for loss to follow-up and non-response bias.

We cannot determine whether cognitive function deviates from the preoperative trend, without multiple preoperative cognitive assessments to establish a trajectory.

\section{BACKGROUND}

Since Bedford's 1955 case series suggesting an association between surgical intervention and cognitive impairment, there has been growing concern among the public that cognitive decline is a common consequence of surgery. ${ }^{1}$ This pervasive view continues to concern patients and their families, fuelled by anecdotal evidence and media coverage. Postoperative cognitive decline (POCD) has been a growing topic for scientific investigation after several studies were published by the International Study of Post-Operative Cognitive Dysfunction group. While several influential studies demonstrated early POCD lasting up to 3 months, the persistence of this cognitive decline has been controversial. ${ }^{2-6}$ Importantly, the population of Americans older than 60 years is projected to double over the next 30 years, ${ }^{7}$ and many will require 
surgery. Acute and long-term cognitive changes related to surgical intervention are particularly distressing to patients and their families. Therefore, clarification of cognitive outcomes would assist patients and practitioners in making an informed decision about the relative risks and benefits of proceeding with elective surgery.

\section{Literature search and review}

A systemised literature review was conducted with the help of a librarian experienced in a systemic review (see Acknowledgements). Ovid Medline was searched for studies published between 1981 and September 2016 using the query: Postoperative or after surgery AND Delirium AND Cognitive function or dementia AND Elderly AND Long-term or intermediate term or follow up or cohort studies or retrospective studies. Articles in English or with English translation were considered. JZC and RT reviewed the 301 search results and found 28 articles that were relevant to our clinical question. Out of the 28 relevant articles, 21 (75\%) showed significant result in the relationship between postoperative delirium (POD) and intermediate-term to long-term cognitive decline (see online supplementary appendix A). These seem to suggest a high pretest probability in studying the ability of POD to predict intermediate-term cognitive decline. However, these studies are heterogeneous in the study design, selected surgical patient population and outcome measurement tools complicating the synthesis of the systemic search to draw conclusions.

POD is a common complication for surgical patients over the age of 60 years. ${ }^{8}$ It is a neurological syndrome characterised by acute and fluctuating disturbances in awareness and attention that represent a change from baseline; in addition to these cardinal features, the diagnosis of delirium requires at least one additional cognitive symptom and the absence of medical conditions that might explain these disturbances. Although usually transient, POD has been associated with several salient adverse outcomes, including mortality, prolonged intensive care unit (ICU) stay and persistent cognitive decline. ${ }^{9-12}$ One prospective cohort study of cardiac surgery patients suggested that patients experiencing POD had prolonged cognitive impairment at 1 year and those with a longer duration of delirium had slower recovery. ${ }^{12}$ However, after adjusting for baseline differences in cognitive function, there was no significant difference compared with controls. The same study also demonstrated that mild cognitive impairment preoperatively may predispose patients to delirium; thus, POD may be exposing patients who are already experiencing subclinical cognitive decline or those who have an underlying vulnerability to cognitive decline. Another study in orthopaedic surgery patients found no association between POD and cognitive decline at 3 months. ${ }^{13}$ While several studies have suggested that POCD can persist past 6 months after surgery, ${ }^{2}{ }^{14}$ others have demonstrated a transient early decline with later recovery. ${ }^{45}$ Furthermore, there is conflicting evidence regarding the long-term cognitive trajectories of postoperative patients, with some suggesting overall improvement and others suggesting overall decline. ${ }^{15} 16$ POD has been posited as a risk factor for lasting decline; however, few articles have addressed the association between POD and persistent POCD (arbitrarily defined as lasting $>6$ months). A prospective matched controlled survey with an average of 30 month follow-up demonstrated that dementia or mild cognitive impairment was diagnosed in $77.8 \%$ of the surviving hip surgery patients with POD, almost doubling the $40.9 \%$ rate of control patients (relative risk=1.9, 95\% CI 1.1 to 3.3). However, the study was underpowered to evaluate the long-term cognitive function, as the power calculation was based on the original study and no intermediate assessments were made to track patients' cognitive function. ${ }^{17}$ Another prospective cohort study followed patients over 36 months and found that patients with POD initially had cognitive decline with a transient recovery, but then exhibited an accelerated pace of long-term cognitive decline when compared with controls. ${ }^{18}$ Overall, the results of these studies suggest an association between POD and persistent cognitive impairment; however, compelling data supporting an independent association are lacking. Many of these studies also do not consider the duration of delirium, which may portend long-term cognitive decline. ${ }^{19} 20$

Pre-existing dementia or cognitive impairment is a known risk factor for $\mathrm{POD},{ }^{8}$ and several studies suggest an association between POD and dementia. One study in elderly orthopaedic surgery patients reported a relative risk of dementia in patient with POD of 10.5 compared with those without POD. ${ }^{21} \mathrm{~A}$ recent study demonstrated a similar association in elderly cardiac surgery patients. ${ }^{22}$ However, it remains unclear whether POD causes, accelerates or signifies an underlying vulnerability to cognitive impairment, and thus further investigation is warranted.

In addition to cognitive outcomes, quality of life and functional capacity are also important patient-centred outcomes that require further investigation. While one study found that elderly surgical patients tended to have long-term cognitive and functional decline compared with non-surgical patients, ${ }^{23}$ few studies have examined the association between cognitive function and quality of life or activities of daily living. One prospective study over a 10-month period demonstrated that delirium was an independent risk factor for becoming dependent for personal activities of daily living after ICU discharge (OR $2.188, \mathrm{P}<0.046)$ and that patients with POD also demonstrated a greater decline in Short-Form-36 domains after discharge, especially in physical function, vitality and social function. ${ }^{24}$ However, this study could not draw conclusions regarding cognitive function due to lack of data. Kastaun et al reported perceived impairment in ability to perform activities of daily living 1 year after surgery, however did not detect significant cognitive decline at that time. ${ }^{25}$ It remains unclear whether cognitive decline results in impaired functional capacity or decreased quality of life. In fact, given the variable definitions of 
POCD, data from previous studies are difficult to interpret. As many of the deficits found in neuropsychiatric testing are subtle, it is possible that detected cognitive impairment has no clinically significant impact on these patient-centred outcomes. Instead of using an arbitrary threshold to dichotomise cognitive function as normal or impaired, it would be more informative to correlate these outcomes with cognitive function as a continuous variable or stratified into multiple groups.

At our institution, patient reported postoperative outcomes are gathered as part of the Systematic Assessment and Targeted Improvement of Services Following Yearlong Surgical Outcomes Surveys (SATISFY-SOS) initiative (NCT02032030). Additionally, the ongoing randomised controlled trial, Electroencephalography (EEG) Guidance of Anesthesia to Alleviate Geriatric Syndromes (ENGAGES) study, is evaluating patients for POD. ${ }^{26}$ Using the scaffold provided by these studies, we aim to identify whether delirium is an independent predictor for intermediate-term cognitive function and quality of life.

\section{Justification}

The population of older adults is projected to double over the next 30 years, and many will undergo elective surgery with the hope of overall improvement in health and quality of life. POD is a common and potentially preventable complication; thus, clarification of its association with persistent declines in cognition and quality of life will inform perioperative care. Prior studies have not demonstrated an association independent of pre-existing patient characteristics and perioperative course. The proposed study addresses the methodological concerns in current literature by: (1) objectively evaluating attention and executive function with validated cognitive tests, (2) obtaining measurements of baseline cognitive function, (3) conducting statistical analysis which includes important potential confounding factors including frailty measures and (4) sampling from an unselected surgical population. Given that delirium is predominantly a disorder of attention and executive function, we will focus our investigation on these cognitive domains. The results of this study will contribute to the broader understanding of postoperative cognitive changes and may help identify and provide therapies for susceptible patients earlier to ultimately improve outcomes.

As part of the ENGAGES study, patients have been enrolling since January 2015, with 804 patients enrolled thus far. Over the last year, the ENGAGES research team has enrolled approximately 35 patients/month, and has gathered baseline cognitive data on a majority of participants. Preliminary screening suggests about $65 \%$ of those enrolled in ENGAGES are eligible for the current study. Anticipating that half of those contacted will enrol, we expect to assess 10-15 patients/month. The scaffold of ENGAGES and trained study members make this substudy highly feasible.
Specific aims

Specific aim 1

Determine whether the incidence and duration of POD predict worse performance on specific cognitive tests at approximately 1 year after surgery.

We hypothesise that POD is independently associated with poorer performance on tests of attention and executive function at 1 year after surgery and that patients with a longer duration of delirium will have more marked cognitive decrement. We will conduct a regression analysis, including likely contributory variables, to assess the association between delirium and cognitive decline at approximately 1 year.

\section{Specific aim 2}

Evaluate whether the incidence and duration of POD predict worse quality of life at approximately 1year postoperatively.

We hypothesise that POD is independently associated with decreased quality of life 1 year after surgery and that patients with a longer duration of delirium will have more marked decrease in health-related quality of life. We will conduct a regression analysis, including likely contributory variables, to assess the association between delirium and health-related quality of life at approximately 1 year.

\section{Specific aim 3}

Explore whether patients who experience POD are more likely to develop dementia within approximately one to 2 years of their surgery.

We hypothesise that POD is independently associated with incident dementia 1-2years after surgery. We will conduct a Cox proportional hazards regression, including likely contributory variables, to assess the rate of incident dementia approximately $1-2$ years after surgery.

\section{STUDY DESIGN}

This prospective cohort study is prespecified substudy of the ENGAGES study. The ENGAGES study is a randomised clinical trial enrolling approximately 1200 patients aged 60 years and older who will undergo elective major surgery at Barnes Jewish Hospital (BJH), St. Louis, Missouri, USA.

The parent trial will recruit patients primarily from the Center for Preoperative Assessment and Planning (CPAP) clinic. Details regarding ENGAGES recruitment and methods have been previously published. ${ }^{26}$ Enrolment in the SATISFY-SOS study, ${ }^{27}$ is an inclusion criteria for participation in ENGAGES. As part of ENGAGES, participants will complete a comprehensive baseline assessment including cognitive tests, frailty measures and screening for delirium and dementia. Postoperatively, patients will have daily evaluations for delirium during the hospital stay. The current study will retrospectively identify 200 participants who reside within approximately 45 miles from the hospital or those who have a planned visit to the hospital between 10 and 16 months 
postoperatively. Patients diagnosed with delirium postoperatively will be compared with those without delirium. A prospective intermediate-term postoperative assessment will be conducted by a research team member blinded to whether the patient developed POD.

\section{STUDY GROUPS}

The target population for this study is patients who underwent preoperative assessment for elective surgery at the CPAP clinic at BJH in St. Louis, Missouri, USA. The ENGAGES study is enrolling 1232 patients who are already enrolled in the STATISFY-SOS study. Inclusion criteria include patients aged 60 years and older, who are competent to provide informed consent and who are undergoing major elective surgery under general anaesthesia with a potent volatile anaesthetic agent that requires a minimum stay of 2 days postoperatively (ie, open cardiac surgery, open thoracic surgery, major vascular surgery, intra-abdominal surgery, open gynaecological surgery, open urological surgery, major orthopaedic surgery, open hepatobiliary surgery and major ear, nose and throat surgery). The ENGAGES study has minimal exclusion, as there are no absolute contraindications to EEG monitoring. Exclusion criteria include neurosurgical procedures, preoperative delirium, patients who are unable to participate adequately in delirium screening, including those who are blind, deaf, illiterate or not fluent in English, patients with a history of intraoperative awareness during intended general anaesthesia and patients who had a second surgery planned within 5 days after the index surgery. This prospective study will enrol 200 patients who are already enrolled in the ENGAGES and SATISFY-SOS studies (see online supplementary appendix B). In addition to previously described inclusion and exclusion criteria, participants will be included in this study if their home residence is $\leq 45$ miles from $\mathrm{BJH}$ or they have a planned visit to the hospital within the specified timeframe. Patients who have not completed the baseline cognitive assessment with Trails A, Trails B and the Stroop Color and Word Test will also be excluded from this study.

\section{RECRUITMENT}

As part of the SATISFY-SOS study, patients have given informed consent to be contacted by Washington University for follow-up of health-related outcomes up to a year after surgery. Eligible participants will be identified by chart review and query of the ENGAGES Research Electronic Data Capture (REDCap) database. A member of the research team will contact potential participants by telephone approximately 10-16 months after surgery for enrolment in this study. Patients may refuse participation in the study. If patients agree to participation, written consent will be obtained when the patient comes in for assessment, prior to any data collection. Patients will then be prospectively evaluated with assessments of cognition, quality of life and frailty measures, which are described in the Data collection section. Meal vouchers will be provided for participation. Patients residing within approximately 45 miles of the hospital, who are willing to participate in the study, but unable or unwilling to come to the study centre will be offered assessment at home by a member of the research team. Additionally, ENGAGES patients who have not completed a 1 year dementia screening will be contacted by telephone approximately 18-24 months after surgery. Phone consent will be obtained to complete dementia screening over the telephone. Participants may refuse to participate.

\section{DATA \\ Data collection}

Routinely obtained baseline assessment in the CPAP clinic includes demographic information, a detailed medical history, surgical history, medications and physical examination. As part of SATISFY-SOS and ENGAGES, baseline assessment using the Barthel Index, Veteran's RAND 12-item Health Survey (VR-12), Eight-item Interview to Differentiate Aging and Dementia, Short Blessed Test (SBT), alcohol sniff test and Personal Health Questionnaire Depression Scale will be performed. Baseline cognitive function will be evaluated by the ENGAGES team with the Trails A and B, and the Stroop Color and Word Test. Some patients may complete computer-based cognitive testing with the National Institutes of Health (NIH) toolbox at baseline (described below). Additionally, frailty will be assessed using grip strength, Timed Up and Go (TUG) test and obtaining fall history at baseline. Participants will have daily delirium assessments by trained researchers with the Confusion Assessment Method in the postoperative period for the duration of their hospital stay. Data collected by the ENGAGES team will be entered into the Washington University School of Medicine REDCap application. ${ }^{18}$ Perioperative data will be retrieved from the hospital's perioperative electronic medical record (EMR; Metavision by iMDsoft, Needham, Massachusetts, USA). High-fidelity perioperative data are routinely captured from our EMR to an SQL server (Microsoft, Redmond, Washington, USA) database. These data include Bispectral Index (BIS) values, EEG burst suppression durations, laboratory data, intraoperative medications, physiological readings and postoperative recovery parameters. All the data for SATISFY-SOS are integrated from various data sources and are stored in a single data repository housed in the Department of Anesthesiology at Washington University. Table 1 contains details regarding variables to be collected for the study.

\section{Cognition}

The primary outcome in this study will be a composite score from three cognitive tests evaluating attention and executive function: Trails A, Trails B and Stroop Color and Word Test. These cognitive tests will be repeated approximately 1 year after surgery to evaluate 


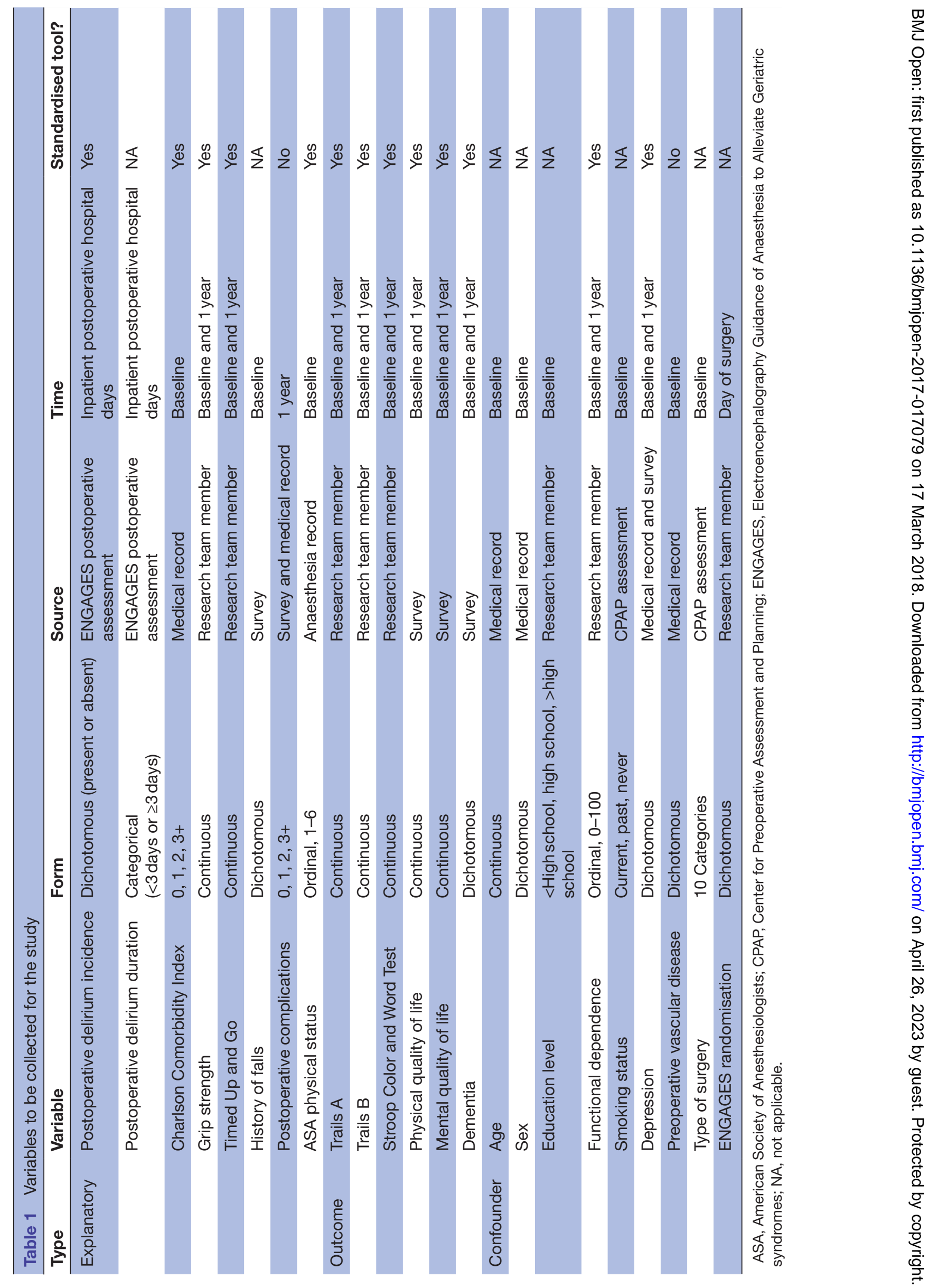


intermediate term cognitive function. The research team member performing the assessments will be blinded to whether the patient developed POD. In addition to the above tests, this study proposes to use the Cognition Battery of the NIH Toolbox Assessment of Neurological and Behavioural Function (http://www.nihtoolbox.org). The NIH Toolbox Cognition Battery (Feinberg School of Medicine, Northwestern University, Chicago, IL), is a comprehensive yet concise standardised computer-based assessment battery; we plan to include tasks to evaluate attention (Flanker Inhibitory Control and Attention Test), episodic memory (Picture Sequence Memory Test) and executive function (Dimensional Change Card Sort Test and Flanker Inhibitory Control and Attention Test). Patients will complete the NIH Toolbox Cognition tests above, which takes approximately $10 \mathrm{~min}$ to complete. The NIH Toolbox Cognition Battery has been validated for use across a wide range of ages. ${ }^{2899}$ Self-reported cognitive function will be evaluated by the Patient Reported Outcomes Measurement Information System (PROMIS). PROMIS, funded by the NIH, is a set of highly reliable, valid, flexible, precise and responsive assessment tools that measure patient-reported health status. PROMIS V.1.0-Applied Cognition-General Concerns-Short Form 4a is comprised four questions about thinking and cognition. PROMIS V.1.0-Applied Cognition-Abilities-Short Form $4 \mathrm{a}$ is comprised four questions about memory. Evaluation for dementia with the SBT will be completed for all patients at baseline, and again between 1 and 2 years after surgery. The SBT has been shown to have 95\% sensitivity for detecting cognitive dysfunction when compared with the Mini-Mental State Examination, and can be administered in approximately $3 \mathrm{~min}$, making it an excellent screening tool. ${ }^{30}$

\section{Quality of life}

As part of the ongoing SATISFY-SOS study, patient self-reported health-related quality of life information will be assessed through the VR-12 at baseline (preoperatively) and also 1 year after surgery. The VR-12 was derived from the Veterans RAND 36 Item Health Survey (VR-36) and contains 12 items relating to quality of life, including physical and mental health, as well as specific questions about functional status. Physical and Mental Health Summary Scores will be calculated. The VR-12 has been validated and is widely applied as a metric for tracking health-related quality of life in the United States. $^{31}$

\section{Functional status}

We will assess functional status with the Barthel index for Activities of Daily Living and Lawton Instrumental Activities of Daily Living Scale. The Barthel Index is a measure of patient performance in 10 activities comprised of eight questions about personal care and two questions about mobility. The Lawton Scale assesses independent living skills in eight areas of function.
Frailty

Information regarding weight loss, endurance and physical activity level are routinely collected in the preoperative clinic and as part of SATISFY-SOS. We will also measure grip strength and the TUG test. Grip strength will be assessed with three measurements in the dominant hand using a Jamar handheld dynamometer (Lafayette Instruments, Lafayette, Indiana, USA). Maximal grip strength will be selected for analysis.

\section{Data management}

A customised database has been developed to facilitate data management for this study using the REDCap system. REDCap is a secure web-based data capture system available to research groups receiving support from the Institute of Clinical and Translational Sciences at Washington University. It is maintained by the Washington University Division of Biostatistics with multiple capabilities to simplify data management and analysis. These include intuitive data entry, data audit and tracking, import of externally sourced data and automated data export for analysis. REDCap requires unique user identification and password combinations for secure access.

\section{STATISTICAL CONSIDERATION}

\section{Sample calculations}

For specific aims 1 and 2, sample size calculations have been performed using G*POWER V.3.0.10. Power analysis for a multiple regression with 15 predictors was conducted to determine a sufficient sample size using an $\alpha$ of 0.05 , a power of 0.9 and a medium effect size $\left(f^{2}=0.1\right)$. Based on the aforementioned assumptions, the desired sample size is 130 patients. We will plan to recruit 200 patients, which will increase the power for the primary outcome and prevent overfitting of the regression model.

For specific aim 3, sample size calculation has been performed using PS V.3.1.2. Based on results of a previously published meta-analysis of four studies investigating the use of BIS-guided anaesthetic administration, we conservatively assume an incidence of POD of $25 \%$. A previous study has reported the median age at the onset of dementia to be approximately 84 years old. ${ }^{32}$ To detect a HR of 2.0 in patients with delirium compared with those without delirium, with $80 \%$ power and $\alpha=0.05$, we will need 516 patients (129 in the delirium group, and 387 in the control group). To account for a $10 \%$ attrition rate, we will recruit 570 patients.

\section{Statistical analysis}

We will perform and report descriptive statistics (mean and SD) for all variables between patients with and without the diagnosis of POD. Descriptive statistics will include $\chi^{2}$ tests for categorical variables, Student's t-test for normally distributed continuous variables and Mann-Whitney U test for non-parametric continuous variables. 


\section{Specific aim 1}

Determine whether the incidence and duration of POD predict worse performance on specific cognitive tests at approximately 1 year after surgery.

The primary endpoint of our analysis is cognitive function 1 year after surgery, as measured by the Trails A and $\mathrm{B}$ and the Stroop Color and Word Test. Z-scores will be calculated for performance for each test and combined to form a composite measure. Multivariable regression analysis will be used to determine whether the development of POD is a predictor variable for cognitive function, independent from other possible contributory factors. POD will be entered as two categorical variables: for incidence (present or absent) and for duration $(<3$ days POD or $\geq 3$ days POD). The following variables will be included in the model: age, sex, American Society of Anesthesiologists (ASA) physical score, and Charlson Comorbidity Index. The following known or suggested risk factors cognitive decline will also be included in the model: baseline cognitive function, education level, type of surgery, randomisation group, preoperative depression, preoperative vascular disease, postoperative complications, composite score on frailty tests and a history of falls. We will use a model with backward elimination to avoid exclusion of predictors with suppressor effects. While cognition will be entered as a continuous variable, we will consider a change of $1 \mathrm{SD}$ in cognitive score as a minimal clinically important difference.

\section{Specific aim 2}

Evaluate whether the incidence and duration of POD predict worse quality of life at approximately 1 year postoperatively.

The primary endpoint of our analysis is quality of life 1 year after surgery, as measured by the change in VR-12 score between preoperative and postoperative assessments. Again, a multivariable regression analysis will be performed to evaluate whether the development of POD is an independent predictor variable for the change in quality of life. POD will be entered as two categorical variables: for incidence (present or absent) and for duration ( $<3$ days POD or $\geq 3$ days POD). The following variables will be included in the model: age, sex, ASA physical score, Charlson Comorbidity Index, type of surgery, randomisation group, preoperative depression, preoperative vascular disease, postoperative complications, cognitive function, composite score on frailty tests and a history of falls. As above, we will use a model with backward elimination, to avoid exclusion of predictors with suppressor effects. Previous work by our group has calculated the minimal clinically important difference for overall VR-12 quality of life to be 1.4 for improvement and 3.6 for deterioration. ${ }^{33}$

For the above aims, we expect this study to be limited by loss to follow-up, estimated to be $50 \%$. Those lost to follow-up are likely to have more severe disease or deterioration in health. In order to determine the influence of missing data in our analysis, we will also conduct a sensitivity analysis. We will calculate the average cognition scores of those who declined. We will repeat the above models including missing subjects, assuming all patients who did not participate had cognitive decline, quantified by the average calculated from participants above. Additionally, to examine whether our sample selection method may bias the results, we will compare baseline characteristics of those ENGAGES patients who were eligible for our study with those who were not eligible.

\section{Specific aim 3}

Explore whether patients who experience POD are more likely to become demented within approximately $1-2$ years of their surgery.

The primary endpoint of our analysis is incident dementia. A Cox proportional hazards regression will be used to explore the likelihood of incident dementia associated with POD. POD will be entered as a dichotomous variable (absent or present). The following variables will be included in the model: age, sex, ASA physical score, Charlson Comorbidity Index, preoperative cognitive function, type of surgery, randomisation group, preoperative depression, preoperative vascular disease, postoperative complications, and composite score on frailty tests and a history of falls.

All statistical analyses will be performed using SAS, V.9.4. All other tests will be two sided. By arbitrary convention, all tests will be two sided and considered statistically significant at $\mathrm{P}<0.05$, and all results will be presented with estimates and $95 \%$ CIs.

\section{Prespecified additional analyses and substudies}

The primary aim of this study is to determine whether POD predicts intermediate-term cognitive impairment. However, as a substudy of ENGAGES and SATISFY-SOS, several other clinically relevant outcomes can be explored as well. SATISFY-SOS systematically collects detailed information regarding patient-centred outcomes, including patient perceived cognition. Correlation between performance on neurocognitive testing and patient perceived cognition would be interesting and useful clinically. Furthermore, the information regarding patients' characteristics will allow exploration of risk factors for intermediate-term cognitive impairment. To investigate effects on memory, we will perform a secondary analysis comparing the results of the SBT. Lastly, as part of ENGAGES, some patients will have baseline NIH Toolbox cognitive testing, allowing description of long-term cognitive trajectories as evaluated by these tests.

\section{Limitations}

This study contains several limitations. First, of most concern is the potential for non-response bias if patients who do not participate are not a random subset of those contacted. We will attempt to minimise the non-response rate by offering to visit patients at their homes if patients are unwilling to come to the study centre. We expect that those lost to follow-up would be more likely to 
have more severe disease or health deterioration, potentially affecting cognitive function. We will thus conduct appropriate sensitivity analyses to determine how missing outcomes data could alter the findings of our study. Second, with an observational study, we cannot establish a causal relationship between POD and persistent declines in cognitive and quality of life. If an independent relationship is found, we shall not be able to disambiguate whether POD in some way causes persistent decline in these outcomes, or whether it is a sign of underlying vulnerability. However, since it is not possible to randomise patients to experience delirium, this limitation might be inevitable. Third, since patients in this study are recruited from a single tertiary care centre, the study sample may not be representative of surgical patients in general. While patients from a tertiary care centre tend to have higher comorbid disease burden than the general surgical population, including comorbidities in the statistical model will account for these differences. Finally, while we have baseline cognitive assessments, we do not have preoperative cognitive trajectories, and thus cannot determine whether the results of cognitive function tests are a deviation from the preoperative trend.

\section{COMPLIANCE}

\section{Subject compliance}

The exposure of this study is postoperative delirium, so no monitoring of exposure compliance is necessary. Methods to improve completion of 1 year cognitive testing are described in the Limitations section.

\section{Withdrawal of subjects}

Subjects may be withdrawn from the study only if requested by the patient. The reason for withdrawal is recorded by the team's clinical project specialist.

\section{Ethical considerations}

Written, informed consent is obtained from all participants. As described in the Study groups section, no special allowances are made for patients who are blind, deaf, illiterate or not fluent in English. Participants may withdraw from the study at any time.

\section{Finance and insurance}

Since this study involves only cognitive testing and surveys, it involves no more than minimal risk to the patients. Finance details, insurance details and cover for negligent and non-negligent harm are, therefore, not relevant in this study.

\section{Reporting and dissemination}

Results of this study will be published in a scientific journal. Participants will only be notified individually if discoveries are made that directly impact their health. We intend to make deidentified individual participant data used to obtain the results reported from this study available to researchers providing a study proposal between 3 months and 5years after article publication. This study protocol, the statistical analysis plan and analytic code will also be available.

Acknowledgements We thank Michelle Doering, a medical librarian at Becker Medical Library at Washington University School of Medicine for developing the systemic search criteria.

Contributors Authorship for this study will be given to key personnel involved in study design, recruitment, data collection and data analysis. There are no publication restrictions, and no professional writers will be involved in the generation of the manuscript. MSA, AA-C, JZC, MRM, RT, TW and EL are responsible for conceptualising study design. AA-C, JZC, RT, MRM, HM and AM were responsible for recruitment, enrolment, data collection and editing the protocol. AA-C, JZC and RT are responsible for drafting the protocol. All authors including AA-C, JZC, MRM, $\mathrm{RT}, \mathrm{AM}, \mathrm{HM}, \mathrm{NL}, \mathrm{TW}, \mathrm{EL}$ and MSA have critically revised the protocol and approved the final version.

Funding Funding for this study was awarded by the Foundation for Anesthesia Education and Research (award reference ID RFG-08/15/2016-Aranake-Chrisinger). Funding for the ENGAGES trial was through a UH2/UH3 mechanism grant awarded by the National Institute on Aging (1UH2AG050312-01). Funding for the SATISFY-SOS study was from a grant awarded by the Barnes-Jewish Hospital Foundation (7937-77) and support provided by the Department of Anesthesiology at Washington University.

Competing interests None declared.

Patient consent Obtained.

Ethics approval This study has been approved by the home institution's Institutional Review Board (IRB ID no 201407128).

Provenance and peer review Not commissioned; externally peer reviewed.

Open Access This is an Open Access article distributed in accordance with the Creative Commons Attribution Non Commercial (CC BY-NC 4.0) license, which permits others to distribute, remix, adapt, build upon this work non-commercially, and license their derivative works on different terms, provided the original work is properly cited and the use is non-commercial. See: http://creativecommons.org/ licenses/by-nc/4.0/

C Article author(s) (or their employer(s) unless otherwise stated in the text of the article) 2018. All rights reserved. No commercial use is permitted unless otherwise expressly granted.

\section{REFERENCES}

1. Bedford PD. Adverse cerebral effects of anaesthesia on old people. Lancet 1955;269:259-64.

2. Moller JT, Cluitmans P, Rasmussen LS, et al. Long-term postoperative cognitive dysfunction in the elderly ISPOCD1 study. ISPOCD investigators. International Study of Post-Operative Cognitive Dysfunction. Lancet 1998;351:857-61.

3. Monk TG, Weldon BC, Garvan CW, et al. Predictors of cognitive dysfunction after major noncardiac surgery. Anesthesiology 2008;108:18-30.

4. Selnes OA, Grega MA, Bailey MM, et al. Cognition 6 years after surgical or medical therapy for coronary artery disease. Ann Neurol 2008;63:581-90.

5. Avidan MS, Searleman AC, Storandt M, et al. Long-term cognitive decline in older subjects was not attributable to noncardiac surgery or major illness. Anesthesiology 2009;111:964-70.

6. Dokkedal U, Hansen TG, Rasmussen LS, et al. Cognitive functioning after surgery in middle-aged and elderly danish twins. Anesthesiology 2016;124:312-21.

7. Projected future growth of the older population. Age http://www.aoa. gov/AoARoot/Aging_Statistics/future_growth/future_growth.aspx (accessed 22 Feb 2016).

8. Whitlock EL, Vannucci A, Avidan MS, et al. Ostoperative delirium. Minerva Anestesiol, 2011:77: 448-56.

9. Gottesman RF, Grega MA, Bailey MM, et al. Delirium after coronary artery bypass graft surgery and late mortality. Ann Neurol 2010;67:338-44.

10. Koster S, Hensens AG, Schuurmans MJ, et al. Consequences of delirium after cardiac operations. Ann Thorac Surg 2012;93:705-11.

11. Bickel H, Gradinger R, Kochs E, et al. High risk of cognitive and functional decline after postoperative delirium. A three-year prospective study. Dement Geriatr Cogn Disord 2008;26:26-31. 
12. Saczynski JS, Marcantonio ER, Quach L, et al. Cognitive trajectories after postoperative delirium. N Engl J Med 2012;367:30-9.

13. Jankowski CJ, Trenerry MR, Cook DJ, et al. Cognitive and functional predictors and sequelae of postoperative delirium in elderly patients undergoing elective joint arthroplasty. Anesth Analg 2011;112:1186-93.

14. Ballard C, Jones E, Gauge N, et al. Optimised anaesthesia to reduce post operative cognitive decline (POCD) in older patients undergoing elective surgery, a randomised controlled trial. PLoS One 2012;7:e37410.

15. Abildstrom H, Rasmussen LS, Rentowl P, et al. Cognitive dysfunction 1-2 years after non-cardiac surgery in the elderly. Acta Anaesthesiol Scand 2000;44:1246-51.

16. Cormack F, Shipolini A, Awad WI, et al. A meta-analysis of cognitive outcome following coronary artery bypass graft surgery. Neurosci Biobehav Rev 2012;36:2118-29.

17. Kat MG, Vreeswijk R, de Jonghe JF, et al. Long-term cognitive outcome of delirium in elderly hip surgery patients. A prospective matched controlled study over two and a half years. Dement Geriatr Cogn Disord 2008;26:1-8.

18. Inouye SK, Marcantonio ER, Kosar CM, et al. The short-term and long-term relationship between delirium and cognitive trajectory in older surgical patients. Alzheimers Dement 2016;12:766-75

19. Gunther ML, Morandi A, Krauskopf E, et al. The association between brain volumes, delirium duration, and cognitive outcomes in intensive care unit survivors: the VISIONS cohort magnetic resonance imaging study $^{*}$. Crit Care Med 2012;40:2022-32.

20. Morandi A, Rogers BP, Gunther ML, et al. The relationship between delirium duration, white matter integrity, and cognitive impairment in intensive care unit survivors as determined by diffusion tensor imaging: the VISIONS prospective cohort magnetic resonance imaging study*. Crit Care Med 2012;40:2182-9.

21. Wacker P, Nunes PV, Cabrita $\mathrm{H}$, et al. Post-operative delirium is associated with poor cognitive outcome and dementia. Dement Geriatr Cogn Disord 2006;21:221-7.

22. Lingehall HC, Smulter NS, Lindahl E, et al. Preoperative cognitive performance and postoperative delirium are independently associated with future dementia in older people who have undergone cardiac surgery: a longitudinal cohort study. Crit Care Med 2017;45:1295-303.

23. Schenning KJ, Murchison CF, Mattek NC, et al. Surgery is associated with ventricular enlargement as well as cognitive and functional decline. Alzheimers Dement 2016;12.

24. Abelha FJ, Luís $\mathrm{C}$, Veiga $\mathrm{D}$, et al. Outcome and quality of life in patients with postoperative delirium during an ICU stay following major surgery. Crit Care 2013;17:R257.

25. Kastaun S, Gerriets T, Schwarz NP, et al. The relevance of postoperative cognitive decline in daily living: results of a 1-year follow-up. J Cardiothorac Vasc Anesth 2016;30:297-303.

26. Wildes TS, Winter AC, Maybrier HR, et al. Protocol for the Electroencephalography Guidance of Anesthesia to Alleviate Geriatric Syndromes (ENGAGES) study: a pragmatic, randomised clinical trial. BMJ Open 2016;6:e011505.

27. Helsten DL, Ben Abdallah A, Avidan MS, et al. Methodologic considerations for collecting patient-reported outcomes from unselected surgical patients. Anesthesiology 2016;125:495-504.

28. Bleck TP, Nowinski CJ, Gershon R, et al. What is the NIH toolbox, and what will it mean to neurology? Neurology 2013;80:874-5.

29. Weintraub S, Dikmen SS, Heaton RK, et al. Cognition assessment using the NIH Toolbox. Neurology 2013;80:S54-S64.

30. Carpenter CR, Bassett ER, Fischer GM, et al. Four sensitive screening tools to detect cognitive dysfunction in geriatric emergency department patients: brief Alzheimer's Screen, Short Blessed Test, Ottawa 3DY, and the caregiver-completed AD8. Acad Emerg Med 2011;18:374-84.

31. Selim AJ, Rogers W, Fleishman JA, et al. Updated U.S. population standard for the veterans RAND 12-item health survey (VR-12). Qual Life Res 2009;18:43-52.

32. Xie J, Brayne C, Matthews FE, et al. Survival times in people with dementia: analysis from population based cohort study with 14 year follow-up. BMJ 2008;336:258-62.

33. Kronzer VL, Jerry MR, Ben Abdallah A, et al. Changes in quality of life after elective surgery: an observational study comparing two measures. Qual Life Res 2017;26:2093-102. 